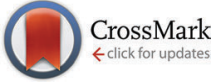

Cite this: Phys. Chem. Chem. Phys., 2015, 17, 11103

Received 9th December 2014, Accepted 4th March 2015

DOI: $10.1039 / c 4 c p 05737 f$

www.rsc.org/pccp

\section{Comment on "How to interpret Onsager cross terms in mixed ionic electronic conductors" by I. Riess, Phys. Chem. Chem. Phys., 2014, 16, 22513}

\author{
Han-Ill Yoo, ${ }^{\star a}$ Manfred Martin ${ }^{b}$ and Juergen Janek ${ }^{c}$
}

\begin{abstract}
Here we show that the Onsager cross terms for ion-electron interactions are not an artifact, but the necessity to phenomenologically and completely describe the mass/charge transport of a mixed ionic-electronic conductor in terms of mobile charged components which are the only experimentally operable species. The use of an appropriate comprehensive defect model may help to reduce the cross terms (which depend on the choice of formal charge of the mobile defects), but it cannot obviate them if long-range Coulombic interactions are in action among the defects.
\end{abstract}

According to irreversible thermodynamics, ${ }^{1-3}$ all the isothermal transport phenomena of a mixed ionic electronic conductor (MIEC), e.g., $\mathrm{A}_{1-\delta} \mathrm{O}_{\nu}$, having one of the ionic components, e.g., $\mathrm{O}^{2-}(=\mathrm{i})$ and holes $\mathrm{h}^{+}(=\mathrm{h})$ as mobile charged components, must be phenomenologically completely described as

$$
\left(\begin{array}{c}
J_{\mathrm{i}} \\
J_{\mathrm{h}}
\end{array}\right)=\left(\begin{array}{cc}
L_{\mathrm{ii}} & L_{\mathrm{ih}} \\
L_{\mathrm{hi}} & L_{\mathrm{hh}}
\end{array}\right)\left(\begin{array}{c}
-\nabla \eta_{\mathrm{i}} \\
-\nabla \eta_{\mathrm{h}}
\end{array}\right)
$$

where $J_{\mathrm{k}}$ and $\eta_{\mathrm{k}}$ denote the flux and electrochemical potential of the mobile charged component $\mathrm{k}(=\mathrm{i}, \mathrm{h})$, respectively. Here, the coupling coefficient matrix $L$ is symmetric, due to Onsager, ${ }^{4}$ or

$$
L_{\mathrm{ih}}=L_{\mathrm{hi}}
$$

Authors $^{5,6}$ often define the ionic charge-of-transport, $\alpha_{i}^{*}$ as

$$
\alpha_{\mathrm{i}}^{*} \equiv\left(\frac{J_{\mathrm{h}}}{J_{\mathrm{i}}}\right)_{\nabla \eta_{\mathrm{h}}=0} \equiv \frac{L_{\mathrm{hi}}}{L_{\mathrm{ii}}}
$$

which phenomenologically corresponds to the number of holes (or electrons) dragged by an anion (or cation) upon its transfer in the absence of their own driving force $\left(\nabla \eta_{\mathrm{h}}=0\right)$. The transport coefficients, and thus also the charge-of-transport, depend on the choice of formal charge number $z_{i}$ of the mobile ions, but their measurable effective charge number $z_{i}+\alpha_{i}^{*}$ is invariant. ${ }^{6}$ In any description of transport in mixed conductors $\mathrm{A}_{1-\delta} \mathrm{O}_{\nu}$, the formal charge of the ionic species is usually chosen on the basis of chemical intuition and experience. In the case of oxygen ions, typically the formal charge number $z_{i}=-2$ is

\footnotetext{
${ }^{a}$ Department of Materials Science and Engineering, Seoul National University, Seoul 151-744, Korea.E-mail: hiyoo@snu.ac.kr

${ }^{b}$ Institute of Physical Chemistry, RWTH Aachen University, 52056 Aachen, Germany ${ }^{c}$ Institute of Physical Chemistry, Justus Liebig-University of Giessen, 35392 Giessen, Germany
}

chosen. Due to interactions with other mobile species the effective charge may differ, which is then properly taken into account by the Onsager cross terms.

Recently, Chatzichrostodoulou et al. ${ }^{7}$ have measured all the $L$-coefficients in the system $\mathrm{Ce}_{0.8} \mathrm{Pr}_{0.2} \mathrm{O}_{2-\delta}$ at $800{ }^{\circ} \mathrm{C}$ by the semiblocking method which was first proposed by Wagner ${ }^{3}$ and first implemented experimentally by Miyatani. ${ }^{8}$ Using the formal charge numbers $z_{\mathrm{i}}=-2$ for oxygen ions and $z_{\mathrm{h}}=+1$ for holes, they have found that $\alpha_{\mathrm{i}}^{*}$ decreases from a value close to +1 to near 0 as the oxygen activity decreases from $\log a_{\mathrm{O}_{2}}=0.0$ to -2.5 .

In a recent paper, Riess ${ }^{9}$ discusses the Onsager cross terms and concludes implicitly that the dependence of the cross terms on the choice of formal charge number makes the cross terms an "artifact". Obviously, this conclusion is incorrect, and in the following we clarify the meaning of the cross terms.

On the work by Chatzichrostodoulou" "and references cited therein":

(1) Riess ${ }^{9}$ first claims that "... in most cases $L_{\mathrm{ih}}=0$. ."

This statement could be misleading. Until now, the attempt to measure $L_{\mathrm{ih}}$ has been made only on nine or so limited numbers of systems and the general conclusion is that $L_{\mathrm{ih}} \neq 0$, the magnitude of which depends on the thermodynamic state of the system, see ref. 3, 8 and 10-18. Even though the mobile ionic defects are fully ionized, $L_{\mathrm{ih}} \neq 0$ if there are long-range interdefect interactions, see below and the Appendix. A correct statement would rather be that $L_{\mathrm{ih}}$ can become small by mathematical transformation if short-range interactions (associations) lead to an ionic defect with a smaller charge number, e.g., the formation of $\mathrm{V}_{\mathrm{O}}^{\bullet}$ by the association of $\mathrm{V}_{\mathrm{O}}^{\bullet \bullet}$ and $\mathrm{e}^{\prime}$, and if this is the majority defect. If the concentrations of $\mathrm{V}_{\mathrm{O}}^{\bullet}$ and $\mathrm{V}_{\mathrm{O}}^{\bullet \bullet}$ are comparable, the cross term becomes appreciable in order to account for the deviation of the effective charge from the integer formal charge, see eqn (4) below. 
(2) Riess $^{9}$ claims that there is "... no explanation for a mutual effect $\left[L_{\mathrm{ih}} \neq 0\right]$. . Indeed there is none."

This claim ignores all of the earlier works on this subject. It is Wagner ${ }^{3}$ who first set up the thermodynamic equations of motion for an MIEC in terms of the mobile charged components, eqn (1), first proposed the experiments to determine the $L$-coefficients, and first considered the physicochemical origin of the mutual effect. He says that "appropriate models of lattice defects are considered in order to see under which condition the cross coefficients may be ignored or yield significant contributions." And he clearly points out that the "finite cross coefficients are due to coupling of transport processes" not only in the short range (i.e., defect association), but also in the long range. For example, as a transfer of $\mathrm{V}_{\mathrm{O}}^{\bullet}$ corresponds to a coupled transfer of $\mathrm{V}_{\mathrm{O}}^{\bullet \bullet}$ and $\mathrm{e}^{\prime}$, one would have $\alpha_{\mathrm{i}}^{*}=1$ if $\mathrm{V}_{\mathrm{O}}^{\bullet}$ and $\mathrm{e}^{\prime}$ were in the majority. Otherwise, one would have to consider the "long-range energetic interaction among charged defects according to Coulomb's law" which "results in a coupling of the motions of the individual defects due to Debye, Hückel, Onsager and Fuoss for aqueous solutions."

Later, Yoo et al. ${ }^{6}$ showed that the cross coefficients include the contributions of any possible defect " $d$ " (structure elements) with different effective charges: in the absence of long-range interactions, one has a non-vanishing charge-of-transport,

$$
\alpha_{\mathrm{i}}^{*}=\frac{\sum_{d} \beta_{d} D_{d} c_{d}}{\sum_{d} D_{d} c_{d}}
$$

if defects with variable charges (due to association) are formed. Here, $\beta_{d}, D_{d}$ and $c_{d}$ stand for the number of electrons (holes) associated with, the self diffusivity of, and the concentration of the defect " $d$ ", respectively. The validity of eqn (4) was then checked to reveal that the cross coefficient is not totally due to short-range interactions or inter-defect associations, but that long-range interactions add up to short-term interactions. ${ }^{6,19}$

In order to rationalize the influence of long range interactions, Janek et al. $^{20,21}$ subsequently applied the Debye-Hückel-OnsagerFuoss theory $^{22,23}$ to solid-state MIECs. The major conclusion is that $\alpha_{i}{ }^{*}$ corresponds to the number of holes or electrons in the electronic charge cloud surrounding the central ionic defect. This may be written for the systems, e.g., $\left\{\mathrm{V}_{\mathrm{O}}^{\bullet \bullet}, \mathrm{e}^{\prime}\right\}$ (as in $\mathrm{CeO}_{2-\delta}$ ) or

$$
\begin{aligned}
& \left\{\mathrm{V}_{\mathrm{Co}}^{\prime \prime}, \mathrm{h}^{\bullet}\right\}\left(\text { as in } \mathrm{Co}_{1-\delta} \mathrm{O}\right) \text { as } \\
& \alpha_{\mathrm{i}}^{*}=\frac{e_{\mathrm{o}}{ }^{2}(1-1 / \sqrt{3})}{6 \pi \varepsilon_{\mathrm{o}} \varepsilon_{\mathrm{r}} k T} \cdot \frac{1}{\kappa^{-1}+a}
\end{aligned}
$$

where $\varepsilon_{\mathrm{o}} \varepsilon_{\mathrm{r}}$ is the dielectric constant of the system, $\kappa^{-1}$ the Debye length, $a$ the distance of the closest approach between an ionic defect and an electron or hole, and the other terms have their usual meanings. The theory has also been extended to systems with more than two defects, e.g. $\left\{\mathrm{V}_{\mathrm{O}}^{\bullet \bullet}, \mathrm{V}_{\mathrm{O}}^{\bullet}, \mathrm{e}^{\prime}\right\} \cdot{ }^{20}$ It has later been found that eqn (5) explains the results more convincingly than eqn (4) for $\mathrm{Co}_{1-\delta} \mathrm{O}^{24}$ and $\mathrm{TiO}_{2-\delta}{ }^{25}$

The present status of understanding is, thus, evenhandedly that the non-vanishing cross effect is partly due to short-range interactions, eqn (4), and partly due to long-range interactions, eqn (5), but that the latter take precedence with increasing defect concentrations. ${ }^{3}$ (Of course, $\lim _{c_{d} \rightarrow 0} \alpha_{\mathrm{i}}{ }^{*}=0$ because then electronic-ionic defect associations are rendered negligible and $\kappa^{-1} \rightarrow \infty$ as well.) In this light, Riess's claim ${ }^{9}$ is incorrect and misleading as well. We would like to add that, for materials with high defect concentrations, even the distinction of shortand long-range interactions becomes arbitrary and the more important consideration becomes that of the phenomenological cross terms. It is necessary to get a complete description of the charge transport.

(3) Riess 9 subsequently states, "It turns out that the appearance of finite cross terms and the ability to measure them are due to an incomplete description of the system at hand." For the system, e.g., $\mathrm{Ce}_{0.8} \mathrm{Pr}_{0.2} \mathrm{O}_{2-\delta}$ for which the ionic defects are known to be $\mathrm{V}_{\mathrm{O}}^{\bullet \bullet}$ and $\mathrm{V}_{\mathrm{O}}^{\bullet}$ in addition to electrons or holes, "if only two," say, $\mathrm{V}_{\mathrm{O}}^{\bullet \bullet}$ and electrons (or holes), "are considered" as charge carriers, "the missing information manifests itself as cross terms $\left(L_{\mathrm{ih}}\right)$ in the theory and leads to misinterpretation of the measurement."

This is a typical misunderstanding arising when one does not properly take into account the detailed inter-relationship between the description of the charged component or buildingunit level, eqn (1), and that of the defect or structure-element level. When the fluxes and forces of all possible structure elements including regular and irregular ones are reduced, in due thermodynamic course, to the independent ones, one eventually ends up with eqn (1) on the charged component level, see the Appendix. All the defect-level information is, thus, condensed into the three independent, measurable coefficients $L_{\mathrm{ii}}, L_{\mathrm{ih}}\left(=L_{\mathrm{hi}}\right)$ and $L_{\mathrm{hh}}$, with no information missing, see ref. 3 and 6 . We would like to note that a comprehensive analysis of transport coefficients, emphasizing the description of the systems with irregular SE or defects of variable charges, but neglecting long range interactions, has also been provided by Maier. $^{27,28}$

It has to be emphasized that the inter-relationships between a measurable transport property and the $L$-coefficients are irrespective of the choice of mobile charged components, e.g., $\left\{\mathrm{O}^{-}, \mathrm{h}^{+}\right\}$instead of $\left\{\mathrm{O}^{2-}, \mathrm{h}^{+}\right\}$. In particular, the effective charge number of mobile ions, $z_{\mathrm{i}}^{*}=z_{\mathrm{i}}+\alpha_{\mathrm{i}}^{*}$, is invariant, in contrast to $z_{\mathrm{i}}$ and $\alpha_{\mathrm{i}}{ }^{*}{ }^{6,19}$ This is because the entropy creation or energy dissipation rate should remain invariant, and hence, the $L$-coefficients are only to be transformed in accordance with this invariance. ${ }^{1,2,19}$ In more practical terms: indeed one can choose the formal charge number of ions such that a short range order is anticipated and the cross terms become smaller, but they will still include long range interactions, which, of course, can be small for dilute defect concentrations.

It is further emphasized that what can be experimentally observed or measured is the transport of the charged components, never that of the structure elements themselves. It is because our system can interact electrochemically with the surroundings only by exchanging charged components or building units $\left(e . g\right.$., $\left.\mathrm{O}^{2-}=\mathrm{O}_{\mathrm{O}}^{\times}-\mathrm{V}_{\mathrm{O}}^{\bullet \bullet}\right)$ : our system can never communicate by exchanging defects or structure elements themselves $\left(\right.$ e.g., $\left.\mathrm{V}_{\mathrm{O}}^{\bullet \bullet}\right)$ due to the structure condition of the system. ${ }^{3,26}$ Therefore, the cross coefficients are a physical necessity and reality 
as well on the level of the charged components, not an artifact arising from a deficient choice of defect carriers.

(4) Riess $^{9}$ finally consummates his argument by saying, "When an appropriate, comprehensive defect model is considered for the MIEC, no cross terms are required."

It is an inherent problem with the defect chemistry and formal description of MIECs that one never knows a priori the "appropriate, comprehensive defect model" for a system under study without observing the related phenomena on the charged component level. Thus, this consummation is just in vain or of no practical significance.

Furthermore, even if such a model were at hand, the cross terms are still required as long as there is a long-range interaction among defects as described by eqn (5), see the Appendix. They are actually unavoidable particularly as defect concentrations increase typically over $10^{-4}$ in mole fractions. ${ }^{3}$ Thus, the author's statement ${ }^{9}$ is incorrect.

It may help the author ${ }^{9}$ to understand the situation better to read Wagner: ${ }^{3}$ if $\alpha_{\mathrm{i}}^{*}$ is known with sufficient precision, then "one obtains additional information for a discussion of the merits of different models of defects to be used in conjunction with other experimental data..."

In conclusion, Riess's interpretation ${ }^{9}$ of the Onsager cross terms is essentially based on the independent migration of differently charged ionic defects and electrons or holes, eventually leading to eqn (4). This interpretation is nothing new because it has already been fully explored, as well as being not totally correct because of the unavoidable long-range interactions among charged defects leading to eqn (5). The careless notion of the cross coefficients as "artifacts" is physically incorrect and will mislead the uninformed reader.

\section{Appendix: Reduction of the transport equations from the structure element level to the charged component level}

Here we will show, by assuming a simple defect structure without sacrificing any generality, how the transport equations on the structure element level are to be reduced to those on the charged component level, eqn (1), in due thermodynamic course.

Suppose that we know a priori the defect structure of a mixed conducting oxide, say $\mathrm{AO}_{1-\delta}$, comprising oxygen vacancies with all possible effective charges and electrons. The mobile structure elements (SE) may then be listed in the Kroger-Vink notation as:

$$
\mathrm{O}_{\mathrm{O}}^{\times}, \mathrm{V}_{\mathrm{O}}^{\times}, \mathrm{V}_{\mathrm{O}}^{\bullet}, \mathrm{V}_{\mathrm{O}}^{\bullet \bullet} ; \mathrm{e}_{\mathrm{C}}^{\prime}, \mathrm{h}_{\mathrm{C}}^{\times}
$$

where particularly $\mathrm{e}_{\mathrm{C}}^{\prime}$ stands for the electrons in the conduction band (C) and $\mathrm{h}_{\mathrm{C}}^{\times}$for the holes in $\mathrm{C}$, the electronic regular $\mathrm{SE}$ corresponding to the ionic regular $\mathrm{SE}, \mathrm{O}_{\mathrm{O}}^{\times}$. These SEs will be henceforth labelled $k=1,2, \ldots, 6$ in order.

Irreversible thermodynamics stipulates that the energy dissipation $(T \sigma)$ due to entropy generation $(\sigma)$ at a certain temperature $(T)$ can be written as

$$
T \sigma=\sum_{k=1}^{6} j_{k} X_{k}
$$

where $j_{k}$ and $X_{k}$ denote the flux and thermodynamic forces $\left(=-\nabla \eta_{k}\right)$ of the SE $k$, respectively. Letting $\ell_{k m}$ denote the coupling transport coefficient on the SE level,

$$
j_{k}=\sum_{m=1}^{6} \ell_{k m} X_{m}
$$

in the linear regime, suggesting that a SE $k$ can be driven to flow by any thermodynamic force $X_{m}$. It is noted that there are 36 $\ell$-coefficients, which are subject to redundancies due to the constraints upon the SE fluxes $j_{k}$ and local quasi-chemical equilibria.

Firstly, they are

$$
\sum_{k=1}^{4} j_{k} \equiv 0 ; \quad \sum_{k=5}^{6} j_{k} \equiv 0
$$

as the numbers of lattice sites and electronic energy states should both be conserved (structure condition). For these to always be true for arbitrary $X_{m}(m=1,2, \ldots, 6)$,

$$
\sum_{k=1}^{4} \ell_{k m} \equiv 0 ; \quad \sum_{k=5}^{6} \ell_{k m} \equiv 0 \quad(m=1-6)
$$

Secondly, the substitution of eqn (A.5) into eqn (A.3) leads to

$$
j_{k}=\sum_{m=1}^{3} \ell_{k m}\left(X_{m}-X_{4}\right)+\ell_{k 5}\left(X_{5}-X_{6}\right)
$$

This means that for $X_{m}=X_{4}(m=1,2,3)$ and $X_{5}=X_{6}, j_{k}=0(k=1-6)$. For this to always be true,

$$
\sum_{m=1}^{4} \ell_{k m} \equiv 0 ; \quad \sum_{m=5}^{6} \ell_{k m} \equiv 0 \quad(k=1-6)
$$

It now turns out that the $36 \ell$-coefficients in eqn (A.3) are subject to 12 inter-relationships in eqn (A.4), and another 12 in eqn (A.7). These 24, however, are not independent of each other at all: as the summations over $m$ of eqn (A.4) and those over $k$ of eqn (A.7) are the same, 4 out of these 24 are rendered redundant, and hence, only 16 out of 36 survive. As these 16 are for the 4 independent fluxes, they further satisfy the Onsager reciprocity $[1,2,4]$ or

$$
\ell_{k m}=\ell_{m k}
$$

Subsequently, we will choose the fluxes of $k=1,2,3$ and 5 as independent only for the sake of convenience.

The thermodynamic forces are also reduced to those of mobile charged components $\mathrm{O}^{2-}(=\mathrm{i})$ and $\mathrm{e}^{-}(=\mathrm{e})$, or holes $\mathrm{h}^{+}$ $(=\mathrm{h})$ via internal quasi-chemical equilibria:

$$
\begin{aligned}
& \mathrm{O}_{\mathrm{O}}^{\times}-\mathrm{V}_{\mathrm{O}}^{\bullet \bullet} \rightleftharpoons \mathrm{O}^{2-}: \quad X_{1}-X_{4}=X_{\mathrm{i}} \\
& \mathrm{e}_{\mathrm{C}}^{\prime}-\mathrm{h}_{\mathrm{C}}^{\times} \rightleftharpoons \mathrm{e}^{-}=-\mathrm{h}^{+}: \quad X_{5}-X_{6}=X_{\mathrm{e}}=-X_{\mathrm{h}} \\
& \mathrm{V}_{\mathrm{O}}^{\times}-\mathrm{V}_{\mathrm{O}}^{\bullet \bullet} \rightleftharpoons 2 \mathrm{e}^{-}=-2 \mathrm{~h}^{+}: \quad X_{2}-X_{4}=2 X_{\mathrm{e}}=-2 X_{\mathrm{h}} \\
& \mathrm{V}_{\mathrm{O}}^{\bullet}-\mathrm{V}_{\mathrm{O}}^{\bullet \bullet} \rightleftharpoons \mathrm{e}^{-}=-\mathrm{h}^{+}: \quad X_{3}-X_{4}=X_{\mathrm{e}}=-X_{\mathrm{h}}
\end{aligned}
$$

By substituting eqn (A.9) into eqn (A.6), one obtains

$$
j_{k}=\ell_{k 1} X_{\mathrm{i}}+\sum_{m=1}^{6} \ell_{k m} \beta_{m} X_{\mathrm{e}}
$$


where the number of electrons associated with the SE $m, \beta_{m}$ takes the numerical values:

$$
\beta_{1}=\beta_{4}=\beta_{6}=0 ; \quad \beta_{2}=2 ; \quad \beta_{3}=\beta_{5}=1
$$

Upon eliminating the dependent fluxes $j_{4}$ and $j_{5}$ due to eqn (A.4) and substituting eqn (A.9) for $X_{m}-X_{4}(m=1,2,3)$ and $X_{5}-X_{6}$, eqn (A.2) takes the form,

$$
T \sigma=j_{1} X_{\mathrm{i}}+\sum_{k=1}^{6} j_{k} \beta_{k} X_{\mathrm{e}}=J_{\mathrm{i}} X_{\mathrm{i}}+J_{\mathrm{e}} X_{\mathrm{e}},
$$

One may, thus, read from eqn (A.12) the fluxes of the mobile charged components, ions $\left(\mathrm{O}^{2-}\right), J_{\mathrm{i}}$, and electrons $\left(\mathrm{e}^{-}\right), J_{\mathrm{e}}$, corresponding to $X_{\mathrm{i}}$ and $X_{\mathrm{e}}$, respectively, as

$$
J_{\mathrm{i}}=j_{1} ; \quad J_{\mathrm{e}}=\sum_{k=1}^{6} j_{k} \beta_{k}
$$

By substituting eqn (A.10) here, one finally obtains the thermodynamic equations of motion on the level of the charged components $\left(\mathrm{O}^{2-}, \mathrm{e}^{-}\right)$, commensurate with eqn (1) in the text $\dagger$,

$$
\left(\begin{array}{c}
J_{\mathrm{i}} \\
J_{\mathrm{e}}
\end{array}\right)=\left(\begin{array}{cc}
L_{\mathrm{ii}} & L_{\mathrm{ie}} \\
L_{\mathrm{ei}} & L_{\mathrm{ee}}
\end{array}\right)\left(\begin{array}{c}
X_{\mathrm{i}} \\
X_{\mathrm{e}}
\end{array}\right)
$$

where the Onsager L coefficients are each expressed in terms of $\ell_{\mathrm{km}}$ as:

$$
\left(\begin{array}{cc}
L_{\mathrm{ii}} & L_{\mathrm{ie}} \\
L_{\mathrm{ei}} & L_{\mathrm{ee}}
\end{array}\right)=\left(\begin{array}{cc}
\ell_{11} & \sum_{m=1}^{6} \ell_{1 m} \beta_{m} \\
\sum_{k=1}^{6} \beta_{k} \ell_{k 1} & \sum_{k=1}^{6} \sum_{m=1}^{6} \beta_{k} \ell_{k m} \beta_{m}
\end{array}\right)
$$

It is noted that eqn (A.8) leads to the Onsager reciprocity on the charged component level, $L_{\mathrm{ie}}=L_{\mathrm{ei}}$ and vice versa.

Let us finally examine the ionic charge-of-transport, $\alpha_{i}^{*}\left(=L_{\mathrm{ie}} / L_{\mathrm{ii}}\right)$. By replacing $\ell_{1 m}$ for the regular SE $k=1$ with those for the irregular structural elements or defects by using eqn (A.5) and (A.7), one gets

$$
\alpha_{i}^{*}=\frac{L_{\mathrm{ie}}}{L_{\mathrm{ii}}}=\frac{-\sum_{k=2}^{4} \sum_{m=2}^{5} \ell_{k m} \beta_{m}}{\sum_{k=2}^{4} \sum_{m=2}^{4} \ell_{k m}}
$$

If, and only if, there were no long-range interactions or $\ell_{k m}=0$ for $k \neq m$, eqn (A.16) takes the form,

$$
\alpha_{i}^{*}=\frac{\sum_{k=2}^{4}\left(-\beta_{k}\right) \ell_{k k}}{\sum_{k=2}^{4} \ell_{k k}}
$$

Noting that $\ell_{k k} \propto D_{k} c_{k}$, one can immediately recognize that eqn (A.17) is nothing but eqn (4) in the text. The ionic charge-oftransport $\alpha_{i}{ }^{*}$ would, thus, phenomenologically correspond to the kinetic average of the number of electrons $\left(\beta_{k}\right)$ or holes $\left(-\beta_{k}\right)$ associated with all kinds of ionic defects, were it not for long-range interactions among defects (i.e., $\ell_{k m} \neq 0$ for $k \neq m$ ).

\section{Acknowledgements}

The authors thank B. Luerssen for creating the TOC graph.

\section{References}

1 S. R. de Groot, Thermodynamics of Irreversible Processes, North-Holland Publishing Company, Amsterdam, 1951.

2 R. E. Howard and A. B. Lidiard, Rep. Prog. Phys., 1964, 27, 161.

3 C. Wagner, Prog. Solid State Chem., 1975, 10, 3.

4 L. Onsager, Phys. Rev., 1931, 37, 405.

5 A. Cukrowski, Acta Phys. Hung., 1989, 66, 159.

6 H.-I. Yoo, H. Schmalzried, M. Martin and J. Janek, Z. Phys. Chem., 1990, 168, 129.

7 C. Chatzichrostodoulou, W.-S. Park, H.-S. Kim, P. V. Hendriksen and H.-I. Yoo, Phys. Chem. Chem. Phys., 2010, 12, 9637.

8 S. Miyatani, Solid State Commun., 1981, 38, 257.

9 I. Riess, Phys. Chem. Chem. Phys., 2014, 16, 22513.

10 D.-K. Lee and H.-I. Yoo, Phys. Rev. Lett., 2006, 97, 255901.

11 N. Ait-Younes, F. Millot and P. Gerdanian, Solid State Ionics, 1984, 12, 431.

12 H.-I. Yoo and M. Martin, Ceram. Trans., 1991, 24, 108.

13 J.-H. Lee and H.-I. Yoo, J. Electrochem. Soc., 1994, 141, 2789.

14 J.-H. Lee, M. Martin and H.-I. Yoo, Electrochemistry, 2000, $68,482$.

15 J.-O. Hong and H.-I. Yoo, J. Mater. Res., 2002, 17, 1213.

16 W.-S. Park, I. Yang and H.-I. Yoo, ECS Trans., 2008, 13, 327.

17 T. Lee and H.-I. Yoo, Solid State Ionics, 2013, 241, 5.

18 H.-S. Kim and H.-I. Yoo, Phys. Chem. Chem. Phys., 2014, 16, 16595.

19 H.-I. Yoo, J.-H. Lee, M. Martin, J. Janek and H. Schmalzried, Solid State Ionics, 1994, 67, 317.

20 J. Janek, M. Martin and H.-I. Yoo, Ber. Bunsen-Ges., 1994, 98, 655.

21 J. Janek and M. Martin, Ber. Bunsen-Ges., 1994, 98, 665.

22 P. Debye and E. Huckel, Phys. Z., 1923, 24, 185; P. Debye and

E. Huckel, Phys. Z., 1923, 24, 305.

23 L. Onsager and R. M. Fuoss, J. Phys. Chem., 1932, 36, 2689. 24 H.-I. Yoo and J.-H. Lee, J. Phys. Chem. Solids, 1996, 57, 65.

25 H.-I. Yoo and D.-K. Lee, Solid State Ionics, 2008, 179, 837.

26 H. Schmalzried, Chemical Kinetics of Solids, VCH, Weinheim, 1995, ch. 2.

27 J. Maier and G. Schwitzgebel, Phys. Status Solidi B, 1982, 113, 535.

28 J. Maier, Z. Phys. Chem., 1984, 140, 191.

$\dagger$ Eqn (1) employs $\mathrm{h}^{+}$, instead of $\mathrm{e}^{-}$. One can easily transform $J_{\mathrm{e}}$ to $J_{\mathrm{h}}$ simply by taking $J_{\mathrm{e}}=-J_{\mathrm{h}}, X_{\mathrm{e}}=-X_{\mathrm{h}}$ and $-\beta_{m}$ as the number of holes associated with the SE $m$. 